IMAGE ARTICLE

\title{
Giant Vegetation in a Mitral Prosthetic Valve
}

\author{
Julio Cesar Sauza-Sosa ${ }^{1,2 *}$ and Nilda Gladys Espinola-Zavaleta ${ }^{1,2}$ \\ ${ }^{1}$ Echo Cardiography Department, National Institute of Cardiology "Ignacio Chavez", Mexico City \\ ${ }^{2}$ Cardiology Department, The American British Cow Dray Medical Centre, Mexico City
}

*Corresponding author: Dr. Julio C Sauza Sosa, Echo Cardiography Department, National Institute of Cardiology "Ignacio Chávez", Juan Badiano \#1, Col. Seccion XVI, ZP 14080, Mexico City, E-mail: sauzamd@hotmail.com

Forty-eight-year-old woman with history of mechanical prosthetic replacement of mitral and aortic valves in February 2005, came to emergency department with fatigue, weakness and chills since two months and dyspnea and edema of lower limbs from three days. The physical examination revealed jugular engorgement, pe- ripheral edema, normal prosthetic clicks and mitral regurgitant murmur II/IV. The laboratory showed anemia, leucocytosis, high NT-pro BNP and high sensitive C-reactive protein. The chest $\mathrm{X}$-ray with cardio megaly and veno capillary pulmonary hypertension (Figure 1). The transesophageal echocardiogram demonstrated giant vegetation in the

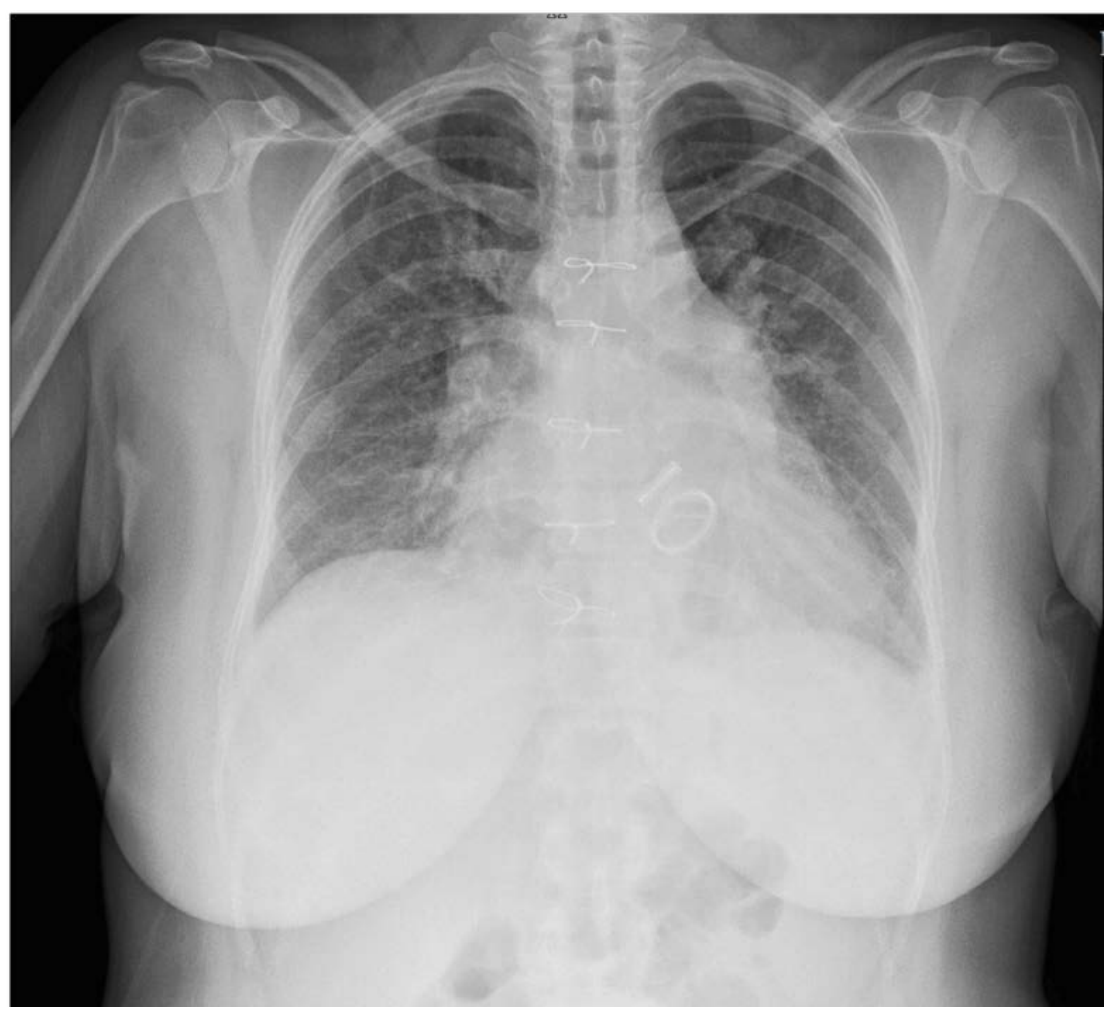

Figure 1: Chest $X$ ray with cardiomegaly and pulmonary veno capillary hypertension. 

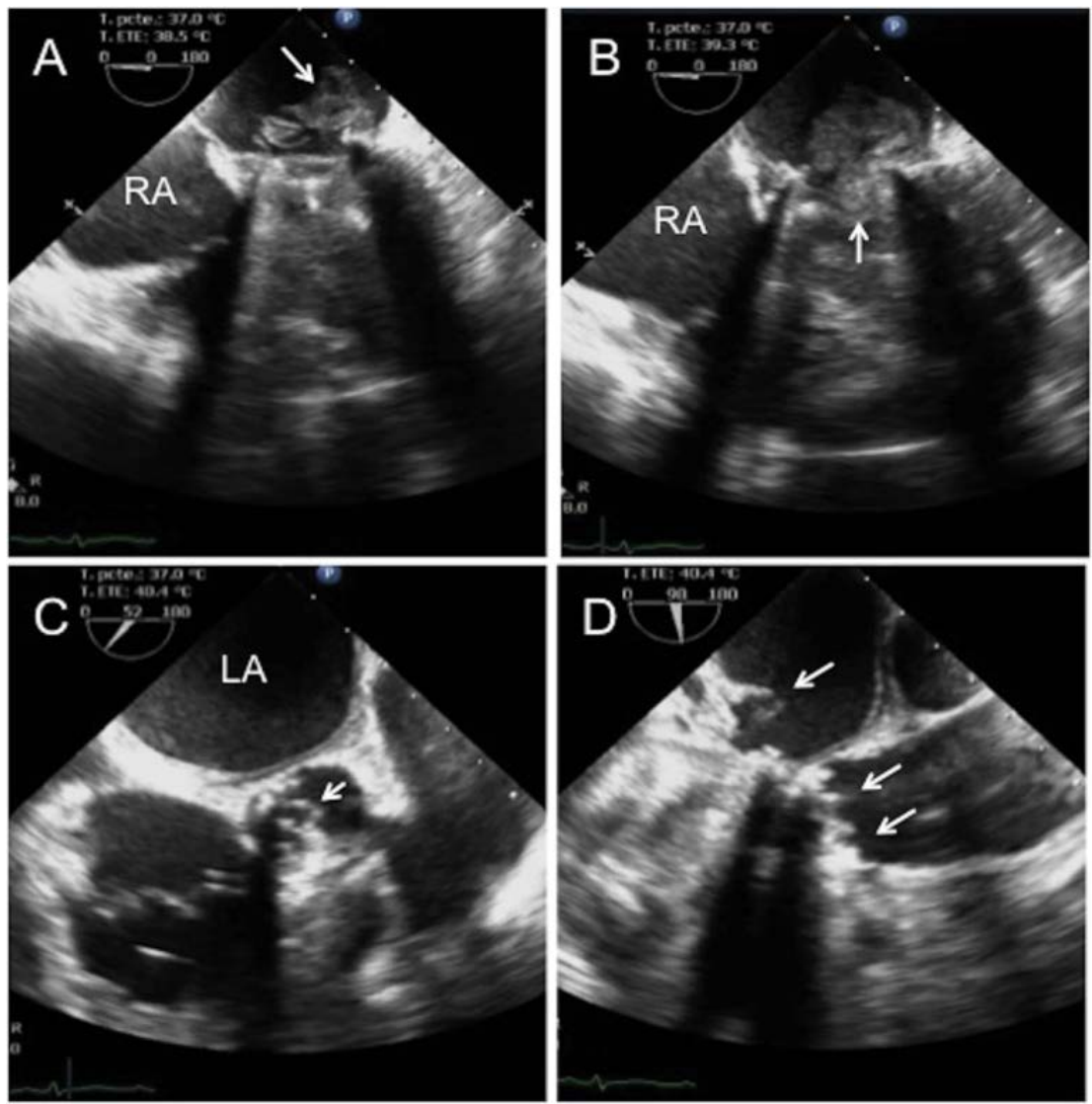

Figure 2: A) Transesophageal four chamber view showing a mechanical prosthetic mitral valve with giant vegetation (white arrow); B) The vegetation is prolapsed into ventricle during diastole; C) Echo cardio graphic view of aortic valve at $52^{\circ}$, with small vegetations pointed by white arrowhead; D) Transesophageal study at $98^{\circ}$ showing the vegetations in mechanical prosthetic aortic valve (white arrows), and prosthetic mitral vegetation in its a trial side (white arrow).

Abbreviations: RA-Right atrium; LA-Left atrium.

trial side of the mitral prosthesis that prolapsed into the ventricle during diastole and small vegetations in prosthetic aortic valve (Figure 2). The blood culture showed streptococcus mitis.

Infective endocarditis is an infection of the endocardial surface of the heart. The diagnosis is based upon positive blood cultures, new valvular murmur, and fever. The transesophageal echocardiography has $85 \%$ sensitivity and $92 \%$ specificity for detection of prosthetic valve vegetations [1].

Prosthetic-valve endocarditis accounts for $7 \%$ to $25 \%$ of infective endocarditis. Early prosthetic-valve endocarditis are usually acquired in the hospital in the first two months after surgery and late cases with more than 12 months are community acquired and the most common etiological agents are streptococcus species (sanguis, bovis, mutans and mitis) $[1,2]$.
The vegetations provoked by streptococcus aureus and candida albicans are generally larger. A strong correlation between size of the vegetation with prognosis and embolic events was found [3]. The echocardiographic findings correlated with surgery; unfortunately the patient died 24 hours later.

\section{References}

1. Mylonakis E, Calderwood SB (2001) Infective endocarditis in adults. N Engl J Med 345: 1318-1330.

2. Rabens RA, Karlson AG, Geraci JE, Jesse Edwards E (1955) Experimental Bacterial Endocarditis Due to Streptococcus Mitis. Circulation 11: 206-214.

3. Leitman M, Dreznik Y, Tyomkin V, Zvi Vered (2011) Vegetation size in patients with infective endocarditis. European Heart Journal Cardiovascular Imaging 13: 330338.

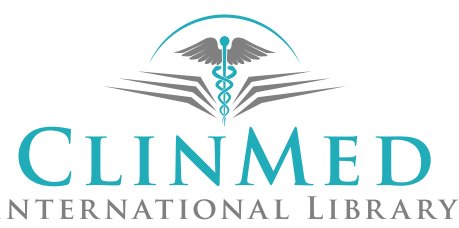

